\title{
PREDOMÍNIO DE MANIFESTAÇÕES RESPIRATÓRIAS NA INDICAÇÃO DE pHMETRIA ESOFÁGICA PROLONGADA EM CRIANÇAS
}

\author{
Helena Ayako Sueno GOLDANI ${ }^{1}$, Themis Reverbel da SILVEIRA ${ }^{1}$, Renata ROCHA ${ }^{1}$, \\ Luciana CELIA ${ }^{1}$, Lucas DALLE MOLLE ${ }^{1}$ e Sergio Gabriel Silva de BARROS ${ }^{2}$
}

RESUMO - Racional - Observa-se um aumento na prevalência de complicações extra-esofágicas do refluxo gastroesofágico. A avaliação do perfil de pacientes submetidos a pHmetria esofágica prolongada poderia auxiliar na identificação de pacientes que poderiam se beneficiar do diagnóstico precoce de refluxo gastroesofágico. Objetivo - Avaliar a população de demanda, indicações e resultados dos exames de pHmetria esofágica prolongada na avaliação de refluxo gastroesofágico em um Serviço de Gastroenterologia Pediátrica. Pacientes e Métodos - Analisaram-se os dados de 190 crianças e adolescentes que realizaram pHmetria esofágica. Realizou-se análise descritiva da população de estudo. As crianças foram categorizadas em cinco grupos conforme a idade. Utilizou-se o aparelho Digitrapper MKIII (Synetics) e software EsopHogram versão 5.7. O parâmetro do exame considerado para análise foi o índice de refluxo. Resultados - As indicações mais freqüentes para o exame foram: asma (26,8\%), apnéia/cianose (20\%), pneumonias de repetição $(18,4 \%)$, síndrome do bebê chiador $(15,8 \%)$ e tosse crônica/rouquidão (11\%). Os grupos etários mais freqüentes nas indicações respiratórias foram: asma - maior de 5 anos (92,2\%); apnéia/cianose - menor de 3 meses (55,3\%); pneumonias de repetição e síndrome do bebê chiador - 3 a 12 meses $(31,4 \%$ e 83,3\%). Os índices de refluxo das crianças com pHmetria positiva para refluxo gastroesofágico foram significativamente mais elevados nas crianças menores de 2 anos, em relação às maiores de 2 anos. Conclus ão - Houve predomínio de manifestações respiratórias nas indicações de pHmetria esofágica. O conhecimento do perfil de pacientes com indicação de pHmetria esofágica pode contribuir para a melhoria da qualidade técnica do exame.

DESCRITORES - Refluxo gastroesofágico. Monitorização fisiológica. Transtornos respiratórios. Crianças.

\section{INTRODUÇÃO}

Refluxo gastroesofágico (RGE) é definido como o retorno involuntário do conteúdo gástrico para o esôfago ${ }^{(19,22)}$.É um dos problemas mais freqüentes dentre os motivos de procura pelos ambulatórios de gastroenterologia pediátrica.

$\mathrm{Na}$ maioria das crianças, os sinais e sintomas de RGE surgem antes do $3^{\circ}$ mês de vida e a maioria apresenta melhora espontânea a partir do $8^{\circ}$ mês de vida, quando a criança inicia a alimentação sólida e assume a postura ereta ${ }^{(24)}$. Este caráter evolutivo do RGE na criança torna-o diferente do adulto ${ }^{(20)}$.

Os lactentes com regurgitação como única manifestação de RGE são considerados portadores de RGE fisiológico. Doença do refluxo gastroesofágico (DRGE) em crianças é a condição de associação com complicações do RGE que podem ser de origem esofágica ou extra-esofágica, tais como esofagite de refluxo, estenose esofágica, esôfago de Barrett, pneumonias de repetição, déficit de crescimento, anemia, síndrome de Sandifer e até morte súbita ${ }^{(19)}$.

Atualmente, observa-se que o RGE está deixando de ser problema restrito ao trato digestório, na medida em que abrange complicações extra-esofágicas. Dentre as novas descrições de complicações do RGE, podem ser citadas a asma brônquica e os problemas otolaringológicos ${ }^{(31)}$, problemas de alimentação ${ }^{(6,10)}$, cólica do lactente ${ }^{(3)}$ e erosão dentária ${ }^{(18)}$. A asma é a complicação mais freqüente com ocorrência de RGE em $47 \%$ até $64 \%$ das crianças asmáticas, embora sem o esclarecimento da relação de causa e efeito ${ }^{(23,25)}$.

Dentre todos os exames disponíveis para o diagnóstico de RGE na criança, a pHmetria intra-esofágica prolongada é o exame com sensibilidade e especificidade acima de $90 \%{ }^{(22)}$. Vários parâmetros podem ser avaliados: número de episódios de refluxo ácido durante as 24 horas, número de episódios de refluxo com duração maior que 5 minutos, duração do episódio mais prolongado e percentagem do tempo com $\mathrm{pH}<4$ em relação ao tempo total de estudo (índice de refluxo). Dentre todos os parâmetros, o índice de refluxo é considerado o mais relevante para o diagnóstico de $\mathrm{RGE}^{(22,30)}$.

Em vista da importância do diagnóstico de RGE nos pacientes com sintomas típicos ou atípicos, o objetivo deste estudo foi avaliar a população de demanda constituída de

${ }^{1}$ Unidade de Gastroenterologia Pediátrica; ${ }^{2}$ Serviço de Gastroenterologia, Hospital de Clínicas de Porto Alegre, Universidade Federal do Rio Grande do Sul, Porto Alegre, RS

Endereço para correspondência: Dra. Helena A. S. Goldani - Prof. André Puente, 200 - apt 501 - 90035-150 - Porto Alegre, RS. E-mail: hegold@uol.com.br 
crianças e adolescentes, suas respectivas indicações e os resultados dos exames de pHmetria intra-esofágica prolongada utilizados na avaliação de RGE em um Serviço de Gastroenterologia Pediátrica.

\section{PACIENTES E MÉTODOS}

Este estudo fez parte do projeto de desenvolvimento "Setor de Motilidade Digestiva do Serviço de Pediatria do Hospital de Clínicas de Porto Alegre", aprovado pelo Comitê de Ética em Pesquisa do Grupo de Pesquisa e Pós-graduação do Hospital de Clínicas de Porto Alegre, Porto Alegre, RS.

Foram analisados os dados de 190 crianças e adolescentes de 0 a 14 anos que realizaram pHmetria intra-esofágica prolongada no Laboratório de Fisiologia Digestiva do Hospital de Clínicas de Porto Alegre, no período de janeiro de 2001 a dezembro de 2003. Os dados foram coletados através de um protocolo padronizado.

\section{pHmetria intra-esofágica prolongada}

Foi utilizado um equipamento Digitrapper MK III (Synetics Medical, Suécia), com sonda de pHmetria com eletrodo de antimônio e outro eletrodo de referência externo (Synetics Medical, Suécia), calibrada em soluções-tampão de pH 1,0 e 7,0. As sondas foram de 1,8 e 2,1 mm de diâmetro (Medtronic) utilizadas, respectivamente, para recém-nascidos e crianças maiores.

Antes de cada procedimento, a sonda foi calibrada com soluções tampão de pH 1,0 e 7,0 (Synetics Medical). Posteriormente, foi introduzida pela via nasal, após jejum de 3 horas para os lactentes e 6 horas para as crianças maiores. $\mathrm{O}$ exame foi realizado de acordo com a padronização internacional proposta pela Sociedade Européia de Gastroenterologia Pediátrica e Nutrição ${ }^{(30)}$, localizando-se o sensor proximal de $\mathrm{pH}$ entre o $8^{\circ}$ e $10^{\circ}$ corpo vertebral torácico e o eletrodo de referência externo sobre o abdome ou na região dorsal.

Durante o exame não houve restrição quanto à dieta, isto é, a criança recebeu a dieta de rotina nos horários habituais. Solicitou-se ao acompanhante o preenchimento de um diário, com anotações referentes aos horários das dietas, posição corporal supina ou ereta e ocorrência de sintomas sugestivos de refluxo gastroesofágico tais como vômito, tosse, chiadeira no peito, apnéia e cianose. Em caso de uso de terapia anti-refluxo prévia, os medicamentos como bloqueadores $\mathrm{H}_{2}$, antiácidos ou agentes procinéticos foram suspensos, pelo menos, 48 horas antes do exame; os inibidores da bomba de próton foram suspensos, pelo menos, 7 dias antes.

Os dados da $\mathrm{pHmetria}$ foram gravados e armazenados no equipamento Digitrapper MK III (Synetics Medical). Ao final de cada exame, os dados foram analisados utilizando-se o software EsopHogram versão 5.7, Gastrosoft Inc. O parâmetro de pHmetria esofágica prolongada considerado para análise foi a percentagem de tempo com $\mathrm{pH}<4$ em relação ao tempo total de estudo (índice de refluxo (IR)). A pHmetria foi considerada positiva para $\mathrm{RGE}$ quando IR $>10 \%$ para crianças menores de 1 ano $^{(28)}$ e IR $>5 \%$ para crianças maiores de 1 ano(4).

Foi realizada uma análise descritiva da população de estudo. Para avaliar a distribuição das indicações de $\mathrm{pHmetria} \mathrm{esofágica} \mathrm{conforme}$ a idade, as crianças foram categorizadas em cinco grupos: grupo 1 (menores de 3 meses), grupo 2 ( 3 a 12 meses completos), grupo 3 (13 a 24 meses completos), grupo 4 ( 25 a 60 meses completos) e grupo 5 (acima de 60 meses até 14 anos). Utilizou-se o teste de Mann-Whitney para a comparação entre os valores dos índices de refluxo positivos das crianças menores e maiores de 2 anos de idade, estabelecendo nível de significância de $P<0,05$.

\section{RESULTADOS}

Do total de 190 pacientes submetidos a pHmetria esofágica prolongada, $102(53,7 \%)$ foram do sexo masculino e $88(46,3 \%)$ do feminino, apresentando discreta predominância do sexo masculino sobre o feminino.

A faixa etária mais freqüente foi a menor de 1 ano de idade (grupos 1 e 2) com 81 pacientes (43\%), sendo 25 (13,2\%) com menos de 3 meses de idade (grupo 1). A segunda faixa etária mais freqüente foi o grupo 5 com 69 pacientes (36,3\%). Seguiram-se os grupos 4 com 21 pacientes $(11,1 \%)$ e grupo 3 com 19 pacientes $(10 \%)$. Dentro de cada faixa etária apresentada, os valores de percentagem de exames de $\mathrm{pHmetria}$ esofágica prolongada positivos para refluxo gastroesofágico foram: grupo $1-52 \%$, grupo 2 $-33,9 \%$, grupo $3-15,7 \%$, grupo $4-38 \%$ e grupo $5-42 \%$.

Houve mais de uma indicação para o exame da pHmetria esofágica prolongada, no entanto, foram incluídas somente as indicações principais do exame. Destas, as quatro indicações mais freqüentes, responsáveis por $81 \%$ delas, foram relacionados com manifestações respiratórias: asma $(26,8 \%)$, apnéias e/ou cianose $(20 \%)$, pneumonias de repetição $(18,4 \%)$ e síndrome do bebê chiador $(15,8 \%)$. O vômito como indicação principal do exame foi observado em somente 14 pacientes $(7,4 \%)$ (Tabela 1). Dentre todos os pacientes, $10(5,5 \%)$ apresentavam paralisia cerebral e 14 (7,3\%) síndrome genética, sendo que destes últimos, 2 possuíam fundoplicatura prévia.

TABELA 1 - Freqüência das principais indicações de pHmetria esofágica prolongada em crianças e exames positivos para refluxo gastroesofágico

\begin{tabular}{lcc}
\hline Indicações & $\mathbf{n}(\%)$ & $\begin{array}{c}\text { pacientes com pHmetria } \\
\text { positiva para RGE }\end{array}$ \\
\hline Asma & $51(26,8 \%)$ & $23 / 51(45 \%)$ \\
Apnéia e/ou cianose & $38(20 \%)$ & $29 / 38(76,3 \%)$ \\
Pneumonias de repetição & $35(18,4 \%)$ & $14 / 35(40 \%)$ \\
Bebê chiador & $30(15,8 \%)$ & $9 / 30(30 \%)$ \\
Tosse e/ou rouquidão & $21(11 \%)$ & $13 / 21(61,9 \%)$ \\
Disfagia & $15(7,8 \%)$ & $7 / 15(46,7 \%)$ \\
Vômito & $14(7,4 \%)$ & $9 / 14(64,2 \%)$ \\
Traqueomalácia & $9(4,7 \%)$ & $2 / 9(33,3 \%)$ \\
Outros* & $3(2,0 \%)$ & $0 / 3(0 \%)$ \\
\hline
\end{tabular}

*controle de terapia anti-ácida, estenose de esôfago operada, pré-fundoplicatura

Os resultados positivos para RGE das pHmetrias esofágicas prolongadas, de acordo com as indicações do exame, estão apresentados na Tabela 1. As indicações com maiores taxas de exames positivos foram: apnéia e/ou cianose (76,3\%), vômito $(64,2 \%)$ e tosse crônica e/ou rouquidão $(61,9 \%)$. A síndrome do bebê chiador $(30 \%)$ e a traqueomalácia $(33,3 \%)$ apresentaram as menores taxas de positividade dos exames.

A distribuição dos grupos etários nas quatro indicações mais freqüentes de pHmetria esofágica prolongada está apresentada 
na Figura 1. Na indicação por asma, a faixa etária mais freqüente foi o grupo $5(92,2 \%)$, na apnéia e/ou cianose foi o grupo 1 $(55,3 \%)$, pneumonias de repetição e síndrome do bebê chiador foi o grupo 2 (31,4\% e $83,3 \%$, respectivamente).

Os valores dos índices de refluxo das crianças menores de 2 anos (mediana 16,3\%) foram significativamente maiores que os das crianças maiores de 2 anos (mediana 8,6\%) $(P<0,01)$.

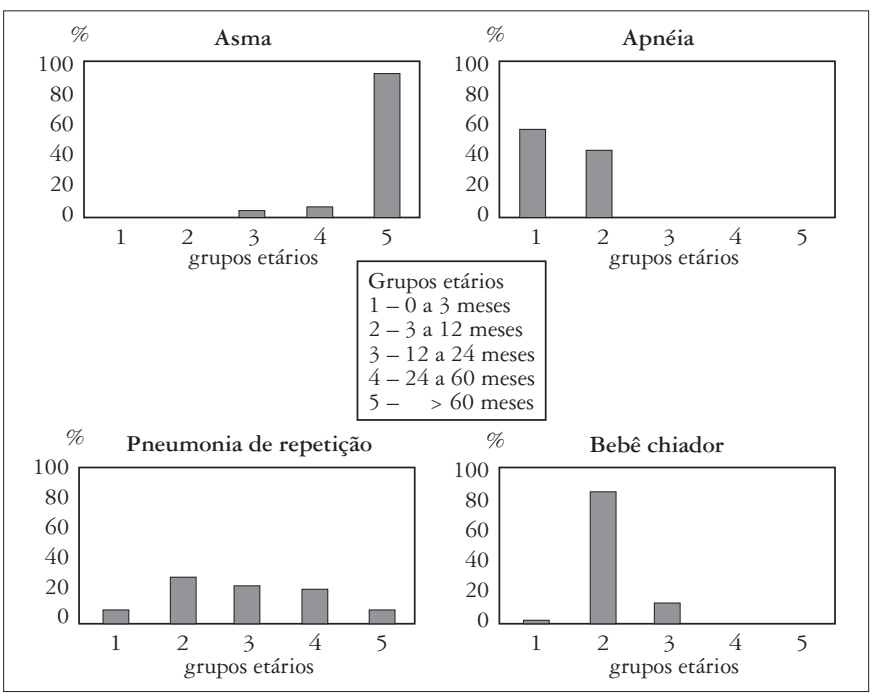

FIGURA 1 - Distribuição percentual dos grupos etários nas quatro indicações mais freqüentes de pHmetria esofágica prolongada em crianças

\section{DISCUSSÃO}

Os dados analisados mostram freqüência maior de crianças menores de 1 ano e predomínio de manifestações respiratórias nas diferentes faixas etárias dos pacientes submetidos ao exame de $\mathrm{pHmetria}$ esofágica prolongada.

Assim como existem estudos de avaliação de demanda para pHmetria esofágica em adultos ${ }^{(17)}$, estes também são pertinentes para o meio pediátrico, visto que o RGE apresenta alta prevalência nesta população. No entanto, apresenta uma forma benigna de evolução na maioria das crianças, não necessitando de exames de investigação ${ }^{(16)}$.

A pHmetria esofágica prolongada pode ser indicada nos casos de suspeita de DRGE e pode ser útil para: a) detectar refluxo oculto nos casos de pneumonias de repetição e broncoespasmos, b) demonstrar relação temporal com sintomas atípicos, como sintomas laríngeos e dor torácica, c) demonstrar a relação com apnéia em estudos polissonográficos, d) avaliar adequação de tratamento clínico em esôfago de Barrett e DRGE grave, e) avaliação pré-fundoplicatura cujos sintomas foram de difícil controle e em pacientes com dúvidas da presença de $\mathrm{RGE}^{(5)}$.

A pHmetria esofágica prolongada é o exame com melhor sensibilidade e especificidade para o diagnóstico do $\mathrm{RGE}^{(15)}$. Apresenta valores de $93 \%$ a $96 \%$, quando comparados com diagnóstico clínico e esofagite histológica ou endoscópica ${ }^{(5)}$. É o único exame que avalia o RGE durante o período de 24 horas, enquanto que os outros disponíveis avaliam somente o RGE pós-prandial como, por exemplo, a radiografia contrastada de esôfago-estômago-duodeno, a cintilografia gastroesofágica e a ecografia abdominal.

Neste estudo monitorizou-se o $\mathrm{pH}$ no esôfago distal, visto que para o diagnóstico de RGE em crianças, a monitorização concomitante do $\mathrm{pH}$ no esôfago proximal não apresenta vantagens em relação à monitorização somente do esôfago distal ${ }^{(2)}$. Para o grupo específico de crianças com problemas respiratórios, ainda não existem recomendações objetivas para a monitorização do pH no esôfago proximal ${ }^{(2)}$.

Algumas limitações do exame poderiam estar associadas a problemas de reprodutibilidade deste, visto que se trata de um fenômeno biológico. Por exemplo, pode ocorrer movimentação da sonda durante o período de estudo, assim como a pHmetria esofágica avalia somente o refluxo de conteúdo ácido. O RGE de conteúdo não-ácido poderia ser avaliado por um exame novo, a impedanciometria elétrica intra-luminal, cujos resultados ainda não padronizados para crianças sinalizam como novo exame útil em crianças ${ }^{(29)}$.

Neste estudo, houve predomínio de manifestações extraesofágicas do RGE nas indicações de pHmetria esofágica prolongada, principalmente relacionadas às complicações respiratórias: asma, apnéia, pneumonias de repetição e síndrome do bebê chiador. ANDZE et al. ${ }^{(1)}$ observaram, entre as indicações de pHmetria, prevalência de $43 \%$ de manifestações respiratórias, porém com apnéia como indicação mais freqüente, seguida de asma.

No Brasil, estudos anteriores avaliaram a prevalência de RGE por meio da $\mathrm{pHmetria}$ esofágica prolongada em populações de crianças prematuras $^{(13)}$ e asmáticas ${ }^{(27)}$. A prevalência de exames positivos para RGE foi semelhante entre crianças com sintomas digestivos $(36,4 \%)$ e respiratórios $(23,1 \%)^{(18)}$. No presente estudo, observou-se que $30 \%$ a $76,3 \%$ dos exames foram positivos para RGE nas crianças com diferentes manifestações respiratórias. Este fato pode ressaltar a importância das complicações extraesofágicas na fisiopatologia do RGE em crianças, visto que já foi observada associação entre episódios de queda de saturação de oxigênio e $\mathrm{RGE}^{(12)}$.

Tomando-se como referência as quatro indicações mais freqüentes, a distribuição das diferentes faixas etárias mostrou algumas características peculiares do RGE em crianças. O grupo de crianças menores de 3 meses foi relacionado com apnéia e/ou cianose, enquanto que os lactentes de 3 a 12 meses com pneumonias de repetição e a síndrome do bebê chiador; e as crianças maiores de 5 anos com a asma. Estes dados refletem a diversidade de manifestações de RGE nas diferentes faixas etárias, reforçando a diferença de manifestação do RGE entre a criança e o adulto ${ }^{(20)}$.

Em relação à idade de todos os pacientes avaliados, observase freqüência maior de crianças menores de 1 ano. Observa-se maior prevalência de RGE em crianças desta faixa etária ${ }^{(16)}$, cuja maturidade do desenvolvimento da motilidade do trato gastrointestinal ainda não atingiu a sua plenitude.

Estima-se que $25 \%$ a $30 \%$ das crianças menores de 1 ano apresentam sibilância ${ }^{(26)}$. Maior prevalência de sintomas da síndrome do bebê chiador seria esperada nesta faixa etária, já que a associação entre RGE e os sintomas respiratórios já foi demonstrada ${ }^{(23)}$. No entanto, é importante ressaltar que ainda não foi estabelecida a relação de causa-efeito entre $\operatorname{ambos}^{(25)}$. 
A fisiopatologia do RGE depende de alguns fatores tais como a pressão basal do esfíncter inferior do esôfago (EIE), o relaxamento transitório do EIE, a depuração esofágica, o esvaziamento gástrico e a pressão intra-abdominal ${ }^{(7,8)}$. Em relação aos sintomas de sibilância, além da influência de todos esses fatores, os sintomas poderiam ser decorrentes da microaspiração do conteúdo gástrico; broncoconstrição via ativação do reflexo vagal, induzido pelo refluxo ácido na mucosa esofágica; ou ainda, estimulação dos neurônios sensitivos com liberação de taquicininas envolvidas no processo de broncoconstrição ${ }^{(25)}$.

O vômito como sintoma principal de RGE também foi indicação para o exame, porém sempre esteve associado a outro fator de gravidade do RGE, por exemplo, a paralisia cerebral. Os sintomas de vômito e regurgitação não acompanhados de manifestações de complicações, não foram indicação para o exame, visto que não necessitam de investigação com pHmetria esofágica prolongada ${ }^{(19)}$.

Os problemas neurológicos estiveram presentes em 5,5\% das indicações e as síndromes genéticas em 7,3\% das indicações para o exame. Excetuando-se o estudo de ANDZE et al. ${ }^{(1)}$, que encontraram $1 \%$ de crianças com problemas neurológicos entre aquelas com manifestações respiratórias de RGE, observa-se falta de estudos com avaliação de população de demanda para pHmetria esofágica. Por outro lado, quando os pacientes são selecionados por doença neurológica, alguns estudos observaram prevalência de até $70 \%$ de RGE nesse grupo de pacientes ${ }^{(9,22)}$. É importante ressaltar que todos os pacientes deste estudo com problemas neurológicos ou síndrome genética, apresentaram sintomas de complicações de RGE, embora a investigação de RGE seja quase sempre indicada devido à alta prevalência de RGE nesses pacientes.

O conhecimento do perfil de pacientes com indicação de pHmetria esofágica prolongada pode contribuir para a melhoria da qualidade do exame. O desenvolvimento de novos materiais, por exemplo, sondas mais flexíveis e com eletrodo de referência interno poderiam ser aperfeiçoados, visando oferecer mais conforto para os pacientes e otimizar a precisão do exame. A monitorização do $\mathrm{pH}$ intra-esofágico sem a sonda de $\mathrm{pHmetria}$ já foi desenvolvida para os adultos.

Os valores de índice de refluxo dos exames considerados positivos para RGE foram significativamente mais elevados nas crianças menores de 2 anos de idade, comparativamente às crianças maiores de 2 anos. Este fato concorda com uma condição já esperada: o RGE é mais prevalente em lactentes, comparativamente às crianças maiores ${ }^{(16)}$ e o limite superior de índice de refluxo de crianças normais é mais elevado em crianças menores de 1 ano $^{(28)}$.

Em relação ao limite superior para índice de refluxo, definiuse $5 \%$ para as crianças maiores de 1 ano, de acordo com estudos bem estabelecidos ${ }^{(4,11)}$, embora alguns serviços utilizem referência de população adulta de $4,2 \%{ }^{(14)}$ até $6 \%{ }^{(15)}$. O índice de refluxo é recomendado como o melhor parâmetro para definição de exame positivo; observa-se, no entanto, falta de padronização de valores nos diversos serviços, visto que os padrões normais estudados foram muito variáveis ${ }^{(15,30)}$. Sob o aspecto de interpretação clínica, a faixa entre $4,2 \%$ a $6 \%$ corresponderia à faixa limítrofe para 0 diagnóstico de RGE, onde a avaliação concomitante dos dados clínicos teria um papel decisivo na conduta do paciente.

Concluindo, observou-se um predomínio de manifestações respiratórias nas indicações de pHmetria esofágica prolongada. $\mathrm{O}$ conhecimento do perfil de pacientes com indicação de pHmetria esofágica poderia contribuir para a melhoria da qualidade técnica do exame.

\section{AGRADECIMENTOS}

Os autores agradecem o apoio do Conselho Nacional de Desenvolvimento Científico e Tecnológico (CNPq), Fundação de Auxílio à Pesquisa do Rio Grande do Sul (FAPERGS) e FIPE - Hospital de Clínicas de Porto Alegre.

Goldani HAS, Silveira TR, Rocha R, Celia L, Dalle-Molle L, Barros SGS. Predominant respiratory symptoms in indications for prolonged esophageal pH-monitoring in children. Arq Gastroenterol 2005;42(3):173-7.

ABSTRACT - Background - An increasing prevalence of extraesophageal complications of gastroesophageal reflux (GER) has been seen. Evaluation of clinical profiles of patients submitted to prolonged esophageal $\mathrm{pH}$ monitoring could help to identify the patients who could benefit from the early diagnosis of GER. Objective - To evaluate the population, indications and results of esophageal $\mathrm{pH}$-monitoring for the diagnosis of GER in a pediatric gastroenterology clinic. Patients and Methods - Data from 190 children and adolescents who had esophageal $\mathrm{pH}$-monitoring were analyzed. A descriptive analysis of the population was performed and the children were categorized in five groups according to the age. The equipment used was a Digitrapper MKIII (Synetics) and all data were analyzed by using a software EsopHogram 5.7. Reflux index was established for analysis. Results - The most frequent indications for the exam were: asthma (26,8\%), apnea/cianosis $(20 \%)$, recurrent pulmonary infections $(18,4 \%)$, wheezing infant syndrome $(15,8 \%)$ and chronic cough/horseness $(11 \%)$. The most frequent groups of age for the respiratory symptoms were: asthma - above 5 years $(92,2 \%)$; apnea/cianosis - under 3 months $(55,3 \%)$; recurrent pulmonary infections and wheezing infant syndrome - 3 to 12 months $(31,4 \%$ and $83,3 \%)$. Reflux index of patients with positive exam for GER was significantly higher in patients under 2 years than above 2 years of age. Conclusion - Respiratory symptoms were predominant among the indications for esophageal pH-monitoring in children. The knowledge of the clinical profile of the patients who had esophageal $\mathrm{pH}$ monitoring could help to improve the technical quality of the exam.

HEADINGS - Gastroesophageal reflux. Monitoring, physiologic. Respiration disorders. Children. 


\section{REFERÊNCIAS BIBLIOGRÁFICAS}

1. Andze GO, Brandt ML, St Vil D, Bensoussan AL, Blanchard H. Diagnosis and treatment of gastroesophageal reflux in 500 children with respiratory symptoms: the value of pH monitoring. J Pediatr Surg 1991;26:295-300.

2. Arana A, Bagucha B, Hauser B, Hegar B, Urbain D, Kaufman L, Vandenplas Y. pH monitoring in the distal and proximal esophagus in symptomatic infants. J Pediatr Gastroenterol Nutr 2001;32:259-64.

3. Berkowitz D, Naveh Y, Berant M. 'Infant colic' as the sole manifestation of gastroesophageal reflux. J Pediatr Gastroenterol Nutr 1997;24:231-3.

4. Boix-Ochoa J, Lafuenta JM, Gil-Vernet JM. Twenty-four hour esophageal pH monitoring in gastroesophageal reflux. J Pediatr Surg 1980;15:74-8.

5. Colletti RB, Christie DL, Orenstein SR. Indications for pediatric esophageal $\mathrm{pH}$ monitoring. Statement of the North American Society for Pediatric Gastroenterology and Nutrition. J Pediatr Gastroenterol Nutr 1995;21:253-62.

6. Douglas JE, Byron M. Interview data on severe behavioral eating difficulties in young children. Arch Dis Child 1996;75:304-8.

7. Goldani HA, Fernandes MI, Vicente YA, Dantas RO. Aspectos fisiopatológicos do refluxo gastroesofágico na infância. Arq Gastroenterol 1996;33:102-114

8. Goldani HA, Fernandes MI, Vicente YA, Dantas RO. Lower esophageal sphibcter reacts against intraabdominal pressure in children with symptoms of gastroesophageal reflux. Dig Dis Sci 2002;47:2544-8.

9. Gustafsson PM, Tibbling L. Gastroesophageal reflux and oesophageal dysfunction in children and adolescents with brain damage. Acta Paediatr 1994;83:1081-5.

10. Hyman PE. Gastroesophageal reflux: one reason why baby won't eat. J Pediatr 1994; 125:103s-9.

11. Mahony MJ, Migliavacca M, Spitz L, Milla PJ. Motor disorders of the esophagus in gastroesophageal reflux. Arch Dis Child 1988;63:1333-8.

12. Meyer R, Fischer GB. Associação entre refluxo gastroesofágico e quedas de saturação transcutânea de oxigênio da hemoglobina em lactentes com doença ventilatória obstrutiva crônica. J Pediatr (Rio J) 2001;77:89-95.

13. Mezzacapa MAMS, Collares EF. Utilização da monitorização prolongada de $\mathrm{pH}$ esofágico no diagnóstico da doença pelo refluxo gastroesofágico em recém-nascidos. J Pediatr (Rio J) 1999; 75:237-43.

14. Moraes-Filho J, Cecconello I, Gama-Rodrigues J, Castro L, Henry MA, Meneghell UG, Quigley E. Brazilian Consensus Group. Brazilian consensus on gastroesophageal reflux disease: proposals for assessment, classification, and management. Am J Gastroenterol 2002;97:241-8.

15. Nelson SP, Chen EH, Syniar GM, Christoffel KK. Prevalence of symptoms of gastroesophageal reflux during infancy. A pediatric-based survey. Arch Pediatr Adolesc Med 1997; 151:569-72.

16. Netzer P, Gut A, Heer R, Gries N, Pfister M, Halter F, Inauen W. Five-year audit of ambulatory 24-hour esophageal $\mathrm{pH}$-manometry in clinical practice. Scan J Gastroenterol 1999;34:676-82.

17. Neufeld CB, Toporovski MS, Magni AM, Martins VJ, Toledo C. Contribuição ao estudo do refluxo gastroesofágico em crianças: correlação entre cortejo de sinais e sintomas clínicos e a prova da pHmetria esofágica de 24 horas. Rev Paul Pediatr 2003;21:143-52.

18. O'Sullivan EA, Curzon ME, Roberts GJ, Milla PJ, Stringer MD. Gastroesophagea reflux in children and its relationship to erosion of primary and permanent teeth. Eur J Oral Sci 1998;106:765-9.
19. Orenstein SR. Infantile reflux: different from adult reflux. Am J Med 1997;103:14$9 \mathrm{~s}$.

20. Orenstein SR. Gastroesophageal reflux disease in children. Gastroenterol Clin North Am 1999;28:947-69.

21. Reyes AL, Cash AJ, Green SH, Booth IW. Gastroesophageal reflux in children with cerebral palsy. Child Care Health Dev 1993;19:109-18.

22. Rudolph CD, Mazur Lj; Liptak GS, Boyle Jt, Colletti RB, Gerson WT, Werlin SL, North American society for pediatric gastroenterology and nutrition. Guidelines for evaluation and treatment of gastroesophageal reflux in infants and children: recommendations of the North American Society for Pediatric Gastroenterology and Nutrition. J Pediatr Gastroenterol Nutr 2001;32:1s-31

23. Sheikh S, Goldsmith LJ, Howell L, Hamlyn J, Eid N. Lung function in infants with wheezing and gastroesophageal reflux. Pediatr Pulmonol 1999;27:236-41.

24. Shepherd RW, Wren J, Evans S, Lander M, Ong TH. Gastroesophageal reflux in children. Clinical profile and outcome with active therapy in 126 cases. Clin Pediatr 1987;26:55-60

25. Sontag SJ. Gastroesophageal reflux and asthma. Am J Med 1997;103:84s-90.

26. Taussig LM. Wheezing in infancy: when is it asthma? Pediatr Pulmonol 1997;16:90-1.

27. Tebet EM. Pesquisa de refluxo gastroesofágico através de pHmetria esofágica de 24 horas em crianças asmáticas [Dissertation]. Ribeirão Preto: Faculdade de Medicina de Ribeirão Preto da Universidade de São Paulo; 2000.

28. Vandenplas Y, Goyvaerts H, Helven R, Sacre L. Gastroesophageal reflux, as measured by 24 -hour $\mathrm{pH}$ monitoring, in 509 healthy infants screened for risk of sudden infant death syndrome. Pediatrics 1991;88:834-40.

29. Wenzl TG, Moroder C, Trachterna M, Thomson M, Silny J, Heimann G, Skopnik H. Esophageal $\mathrm{pH}$ monitoring and impedance measurements: a comparison of two diagnostic tests for gastroesophageal reflux. J Pediatr Gastroenterol Nutr 2002;34:519-23.

30. Working Group of the European Society of Pediatric Gastroenterology and Nutrition. A standardized protocol for the methodology of esophageal $\mathrm{pH}$ monitoring and interpretation of the data for the diagnosis of gastroesophageal reflux. J Pediatr Gastroenterol Nutr 1992;14:467-71.

31. Yellon RF. The spectrum of reflux-associated otolaryngologic problems in infants and children. Am J Med 1997;103:125s-9s. 